\title{
NOVA METODOLOGIA DE AVALIAÇÃO DA REAÇÃO DE GENÓTIPOS DE CAPIM-COLONIÃO AO ALUMÍNIO'
}

\author{
ANTONIO CARLOS DE OLIVEIRA², JOSÉ ALFREDO USBERTI FILHO e WALTER JOSÉ SIQUEIRA $^{4}$
}

\begin{abstract}
RESUMO - Foi definida nova metodologia de avaliação de genótipos quanto à reação ao alumínio $\left(\mathrm{Al}^{3+}\right)$, presente em soluções nutritivas, através da associação de análises de componentes principais e de estabilidade/adaptabilidade fenotípicas. Os experimentos foram instalados em delineamentos de blocos ao acaso, em parcelas subdivididas, com três repetições e conduzidos em casa de vegetação da Seção de Genética - Centro Experimental de Campinas/IAC, no período de 1995 a 1997. Em diferentes genótipos de capim-colonião (Panicum maximum Jacq.) foram avaliados caracteres de sistema radicular e de parte aérea, sob três concentrações de $\mathrm{Al}^{3+}$ (zero, 12 e $24 \mathrm{mg} / \mathrm{L}$ ) e três períodos de permanência em solução ( 15,30 e 45 dias). Reduções porcentuais, causadas pela presença do $\mathrm{Al}^{3+}$, foram calculadas para cada caráter analisado e, a partir delas, efetuadas as análises acima citadas. Considerável precisão foi observada na diferenciação dos genótipos quanto ao estresse, sugerindo o seu emprego em programas de melhoramento genético.
\end{abstract}

Termos para indexação: Panicum maximum, híbridos, solução nutritiva, fenótipos, estabilidade, adaptabilidade fenotípica, análise multivariada, métodos de melhoramento.

\section{NEW METHODOLOGY OF ALUMINUM RESISTANCE EVALUATION IN GUINEA GRASS}

\begin{abstract}
A new methodology of genotype screening as to aluminum $\left(\mathrm{Al}^{3+}\right)$ reaction has been proposed, based on the association of principal component and stability/adaptability analyses. Experimental trials, using nutritive solutions, have been carried out in randomized complete block designs (split-plot type), with three replications, at Genetics Department in Instituto Agronômico, Campinas, SP, Brazil, from 1995 to 1997. Root system and plant traits have been evaluated in several guinea grass (Panicum maximum Jacq.) genotypes under three $\mathrm{Al}^{3+}$ concentrations (zero, 12 and $24 \mathrm{mg} / \mathrm{L}$ ), during three time periods (15, 30 and 45 days). Percentual reductions, due to the presence of $\mathrm{Al}^{3+}$, have been calculated for each character and analyses performed on them. A good precision has been achieved in genotype differentiation, strongly suggesting the use of the new methodology in plant breeding programs.
\end{abstract}

Index terms: Panicum maximum, hybrids, nutrient solutions, phenotypes, stability, phenotypic adaptability, multivariate analyses, breeding methods.

\footnotetext{
${ }^{1}$ Aceito para publicação em 11 de janeiro de 2000.

Extraído da dissertação de mestrado do primeiro autor, apresentada à Universidade Estadual de Campinas (Unicamp). Projeto auxiliado pela FAPESP.

${ }^{2}$ Biól., M.Sc., doutorando, Dep. de Genética e Evolução, Unicamp, Caixa Postal 6109, CEP 13081-970 Campinas, SP. E-mail: ancaol@zipmail.com.br

${ }^{3}$ Eng. Agrôn., Ph.D., Centro de Genética, Biologia Molecular e Fitoquímica, Instituto Agronômico (IAC), Caixa Postal 28, CEP 13001-970 Campinas, SP. Bolsista do CNPq. E-mail: usberti@cec.iac.br

${ }^{4}$ Eng. Agrôn., Dr., IAC. E-mail: walter@mandic.com.br
}

\section{INTRODUÇÃO}

A toxicidade do alumínio é o principal fator limitante ao estabelecimento de diferentes culturas em solos ácidos (Foy, 1984). No Brasil, a nova fronteira agrícola está localizada, principalmente, em solos de Cerrados, possuidores de elevados teores do elemento. A neutralização do $\mathrm{Al}^{3+}$, por meio do emprego de corretivos em larga escala, é, na maioria dos casos, economicamente inviável pelo custo da aquisição e transporte. 
Dessa maneira, a seleção de genótipos resistentes ao $\mathrm{Al}^{3+}$, em diversas espécies vegetais, é a alternativa mais racional e barata para a solução do problema (Lafever, 1981; Foy, 1983).

Diferentes genótipos podem comportar-se de maneira distinta quanto à reação ao $\mathrm{Al}^{3+}$, tanto no campo como em vasos ou em soluções nutritivas. Tal comportamento diferencial pode ocorrer entre gêneros (McCormick \& Steiner, 1978), entre espécies (McLean \& Gilbert, 1927; Nowak \& Friend, 1995), e mesmo entre indivíduos da mesma espécie (Hernani, 1980; Bastos, 1981; Camargo \& Oliveira, 1981; Furlani \& Clark, 1981; Sivaguru \& Paliwal, 1993).

Os métodos atuais de avaliação de resistência/ tolerância ao $\mathrm{Al}^{3+}$, relatados na literatura, baseiamse em comparações entre genótipos, ou entre genótipos e controles previamente escolhidos. $\mathrm{Em}$ ambos os casos, o efeito do $\mathrm{Al}^{3+}$ não é isolado, sendo os resultados mascarados pelo componente genético de cada material em estudo. Assim, uma metodologia eficiente e racional deveria, em primeiro lugar, isolar o efeito do $\mathrm{Al}^{3+} \mathrm{e}$, em seguida, mediante análises adequadas, promover a discriminação confiável dos genótipos quanto à reação ao estresse.

Em capim-colonião (Panicum maximum Jacq.), importante gramínea forrageira tropical, amplamente utilizada no Brasil, são raros os estudos sobre o comportamento de genótipos em presença do $\mathrm{Al}^{3+}$. Podem ser citadas avaliações de acessos (Thomas \& Lapointe, 1989) e a obtenção de linhagens tolerantes (Hutton, 1989).

Este trabalho descreve a associação de análises multivariada e de estabilidade e adaptabilidade fenotípicas como um método inédito e eficiente de classificação da reação de genótipos ao estresse $\mathrm{Al}^{3+}$, sem o emprego de controles comparativos.

\section{MATERIAL E MÉTODOS}

Foram avaliados 15 híbridos $\mathrm{F}_{1}$ apomíticos e três cultivares comerciais de capim-colonião, cujas genealogias são apresentadas na Tabela 1.

As plântulas de cada genótipo foram obtidas da germinação de sementes em caixas de semeadura e transferidas para as soluções nutritivas no estádio de 3-4 folhas.

Utilizaram-se caixas de plástico de $53 \times 43 \mathrm{~cm}$ e profundidade de $12 \mathrm{~cm}$, com capacidade de 13 litros de solução nutritiva. As plântulas foram dispostas nessas caixas
TABELA 1. Genealogia dos híbridos $F_{1}$ apomíticos e cultivares de capim-colonião avaliados.

\begin{tabular}{ll}
\hline Híbrido/cultivar & Genealogia $^{1}$ \\
\hline H10 & LST-1 SEA-2 \\
H12 & LST-1 x SEA-2 \\
H13 & LST-1 x cv. IAC-Tobiatã \\
H21 & LST-2 x PI-277915 \\
H31 & LST-2 x colonião "Dr. Schanks" \\
H33 & LST-2x colonião "Dr. Schanks" \\
H38 & LST-2 x cv. Aruana \\
H42 & LST-2 $\times$ cv. Aruana \\
H54 & LST-2 x cv. IAC-Centauro \\
H55 & LST-2 $\times$ K-68 \\
H56 & LST-2 $\times$ K-68 \\
H22 & LST-2 $\times$ Híbrido-30 \\
H64 & LSC-1 x Híbrido-30 \\
H79 & LSC-2 $\times$ K-68 \\
H140 & LSC-7 x cv. Aruana \\
IAC-Centauro & LS40 x cv. Katerere \\
IAC-Centenário & LS28 $\times$ cv. Angola \\
IAC-Tobiatã & linhagem K-187 \\
\hline
\end{tabular}

${ }^{1}$ LST-1: linhagem sexual Tobiatã-1; LST-2: linhagem sexual Tobiatã-2; LSC-1: linhagem sexual Centauro-1; LSC-2: linhagem sexual Centauro-2; LSC-7: linhagem sexual Centauro-7; LS40: linhagem sexual $\mathrm{n}^{\circ} 40$; LS28: linhagem sexual $\mathrm{n}^{\circ} 28$.

em placas de isopor de $2,5 \mathrm{~cm}$ de espessura, perfuradas em 60 pontos, eqüidistantes. Cada orifício da placa foi tampado por um disco de espuma com uma cavidade central, através da qual eram colocadas as plântulas, de modo que os sistemas radiculares alcançassem a solução nutritiva.

As soluções nutritivas foram aeradas, ininterruptamente, por períodos de quatro horas, intercalados por um período de duas horas, sem aeração.

A solução nutritiva padrão empregada foi semelhante à definida por Furlani \& Clark (1981), cuja composição é apresentada na Tabela 2.

Foram utilizadas três concentrações de $\mathrm{Al}^{3+}$ (zero, $12 \mathrm{e}$ $24 \mathrm{mg} / \mathrm{L}$ ), as duas últimas adicionadas à solução nutritiva sob a forma de sulfato de alumínio-potássico dodecaidratado $\left[\mathrm{AlK}\left(\mathrm{SO}_{4}\right)_{2} .12 \mathrm{H}_{2} \mathrm{O}\right]$.

As soluções nutritivas foram preparadas com água destilada estéril, seus pH's ajustados, inicialmente, para 4,0 (adição de $\mathrm{HCl} 1 \mathrm{~N}$ ) e monitorados, periodicamente, durante o experimento, sendo trocadas semanalmente.

Durante a transferência das plântulas, foi medido o comprimento inicial de raiz (CIR, em $\mathrm{cm}$ ), tomado do colo da plântula à extremidade da raiz. Decorridos 15,30 e 45 dias de permanência nas soluções nutritivas, as plântulas foram avaliadas quanto às seguintes características: a) altura de plântula (AP, em cm): medida do colo da plântula à extremidade da folha mais alta; b) comprimento de lâmina 
TABELA2. Composição das soluções-estoque e da solução nutritiva empregada ${ }^{1}$.

\begin{tabular}{|c|c|c|c|c|c|c|c|}
\hline \multicolumn{4}{|c|}{ Solução-estoque } & \multicolumn{4}{|c|}{ Solução nutritiva } \\
\hline № & Sal & $\begin{array}{l}\text { Quantidade } \\
(\mathrm{g} / \mathrm{L})\end{array}$ & $\begin{array}{c}\text { Quant. adicion. à } \\
\text { sol. nutritiva } \\
\text { (mL/L) }\end{array}$ & Cátion & $(\mathrm{mg} / \mathrm{L})$ & Ânion & $(\mathrm{mg} / \mathrm{L})$ \\
\hline \multirow[t]{2}{*}{1} & $\mathrm{Ca}\left(\mathrm{NO}_{3}\right)_{2} \cdot 4 \mathrm{H}_{2} \mathrm{O}$ & 270,0 & 0,61 & $\mathrm{Ca}$ & 28,20 & $\mathrm{NO}_{3}-\mathrm{N}$ & 19,76 \\
\hline & $\mathrm{NH}_{4} \mathrm{NO}_{3}$ & 33,8 & & $\mathrm{NH}_{4}-\mathrm{N}$ & 3,40 & $\mathrm{NO}_{3}-\mathrm{N}$ & 3,40 \\
\hline \multirow[t]{3}{*}{2} & $\mathrm{KCl}$ & 18,6 & 0,46 & $\mathrm{~K}$ & 4,44 & $\mathrm{Cl}$ & 4,02 \\
\hline & $\mathrm{KNO}_{3}$ & 24,6 & & $\mathrm{~K}$ & 4,07 & $\mathrm{NO}_{3}-\mathrm{N}$ & 1,47 \\
\hline & $\mathrm{K}_{2} \mathrm{SO}_{4}$ & 44,0 & & K & 8,29 & $\mathrm{SO}_{4}-\mathrm{S}$ & 3,44 \\
\hline 3 & $\mathrm{Mg}\left(\mathrm{NO}_{3}\right)_{2} \cdot 6 \mathrm{H}_{2} \mathrm{O}$ & 142,4 & 0,31 & $\mathrm{Mg}$ & 4,19 & $\mathrm{NO}_{3}-\mathrm{N}$ & 4,85 \\
\hline 4 & $\mathrm{KH}_{2} \mathrm{PO}_{4}$ & 17,6 & 0,05 & $\mathrm{~K}$ & 0,25 & $\mathrm{H}_{2} \mathrm{PO}_{4}-\mathrm{P}$ & 0,20 \\
\hline \multirow[t]{2}{*}{5} & $\mathrm{FeSO}_{4} \cdot 7 \mathrm{H}_{2} \mathrm{O}$ & 24,9 & 0,15 & $\mathrm{Fe}$ & 1,34 & $\mathrm{SO}_{4}-\mathrm{S}$ & 0,78 \\
\hline & $\mathrm{Na}_{2}$ EDTA & 29,6 & & $\mathrm{Na}$ & 0,54 & EDTA & 3,89 \\
\hline \multirow[t]{5}{*}{6} & $\mathrm{MnCl}_{2} \cdot 4 \mathrm{H}_{2} \mathrm{O}$ & 2,34 & 0,15 & $\mathrm{Mn}$ & 0,09 & $\mathrm{Cl}_{2}$ & 0,12 \\
\hline & $\mathrm{H}_{3} \mathrm{BO}_{3}$ & 2,04 & & & & $\mathrm{BO}_{3}-\mathrm{B}$ & 0,05 \\
\hline & $\mathrm{CuSO}_{4} .5 \mathrm{H}_{2} \mathrm{O}$ & 0,20 & & $\mathrm{Cu}$ & 0,007 & $\mathrm{SO}_{4}-\mathrm{S}$ & 0,0036 \\
\hline & $\mathrm{ZnSO}_{4} \cdot \mathrm{H}_{2} \mathrm{O}$ & 0,88 & & $\mathrm{Zn}$ & 0,03 & $\mathrm{SO}_{4}-\mathrm{S}$ & 0,014 \\
\hline & $\mathrm{Na}_{2} \mathrm{MoO}_{4} \cdot 2 \mathrm{H}_{2} \mathrm{O}$ & 0,26 & & $\mathrm{Na}$ & 0,0074 & $\mathrm{MoO}_{4}-\mathrm{Mo}$ & 0,015 \\
\hline
\end{tabular}

${ }^{1}$ Composição final (mg/L): 28,2 de Ca; 17,05 de K; 4,19 de Mg; 29,48 de $\mathrm{NO}_{3}-\mathrm{N} ; 3,4$ de $\mathrm{NH}_{4}-\mathrm{N} ; 3,9$ de S; 0,2 de P; 0,75 de Fe; 4,2 de Cl; 0,09 de Mn; 0,05 de B; 0,007 de $\mathrm{Cu} ; 0,03$ de $\mathrm{Zn} ; 0,54$ de Na; 4,44 de EDTA; 0,015 de Mo; e adição de AlK $\left(\mathrm{SO}_{4}\right)_{2} \cdot 12 \mathrm{H}_{2} \mathrm{O}$ (PM = 474,38) para obter as concentrações de 12 e $24 \mathrm{mg} / \mathrm{L}$ de $\mathrm{Al}^{3+}$.

foliar (CLF, em cm): medida da base ao ápice da maior folha; c) comprimento total de raiz (CTR, em $\mathrm{cm}$ ): medida do colo da plântula à extremidade da raiz; d) comprimento líquido de raiz (CLR, em cm): calculado pela subtração do CIR do valor do CTR; e) reduções porcentuais de altura de plântula e de comprimento líquido de raiz (RPAP e RPCLR) (isolamento do efeito do $\mathrm{Al}^{3+}$ ) calculadas através da fórmula seguinte:

$\mathrm{RP}=1-\left[\frac{\overline{\mathrm{X}}_{\mathrm{p} \cdot \mathrm{Al} \mathrm{l}^{3+}}}{\overline{\mathrm{X}}_{\mathrm{a} \cdot \mathrm{Al} \mathrm{l}^{3+}}}\right] .100$, onde:

$\mathrm{RP}$ : redução porcentual da característica; $\overline{\mathrm{X}}_{\mathrm{p} \cdot \mathrm{Al}^{3+}}:$ média estimada da característica, em presença de $\mathrm{Al}^{3+} ; \overline{\mathrm{X}}_{\mathrm{a} . \mathrm{Al}} \mathrm{l}^{3+}$ : média estimada da característica, na ausência de $\mathrm{Al}^{3+}$.

O delineamento experimental utilizado foi o de blocos ao acaso, em parcelas subdivididas, com três repetições. As concentrações de $\mathrm{Al}^{3+}$ foram alocadas na parcela e os genótipos na subparcela, dispostos aleatoriamente dentro de cada nível de $\mathrm{Al}^{3+}$. Adotou-se o teste de Duncan $(\mathrm{P}=0,05)$ para a comparação entre médias. Dados porcentuais das relações quantitativas RPAP e RPCLR foram transformados em arco seno $\sqrt{\%}$ (Steel \& Torrie, 1980), previamente à execução da análise de variância e do teste de comparação de médias. A análise de variância e os testes de médias das outras características avaliadas foram realizados com os dados originais.

Foi realizada análise de componentes principais (ACP) dos dados para a diferenciação dos genótipos quanto à reação à toxidez de $\mathrm{Al}^{3+}$, empregando-se as características e relações quantitativas citadas anteriormente. Considerando-se as três concentrações de $\mathrm{Al}^{3+}$ empregadas como ambientes distintos, avaliaram-se a estabilidade e a adaptabilidade dos híbridos/cultivares frente ao estresse, com base em médias do caráter AP, utilizando-se o modelo genético-estatístico de Eberhart \& Russell (1966).

\section{RESULTADOS E DISCUSSÃO}

A combinação de fatores (período de permanência em solução e concentração de $\mathrm{Al}^{3+}$ ) que melhor discriminou os materiais genéticos em estudo foi a de 45 dias e de $24 \mathrm{mg} / \mathrm{L}$. Diferenças altamente significativas foram observadas tanto entre os genótipos (dentro de cada concentração de $\mathrm{Al}^{3+}$ ) como entre as concentrações (para cada genótipo) com respeito às características de parte aérea e de sistema radicular e as reduções porcentuais (Tabelas 3 e 4).

Reduções significativas de AP foram detectadas em todos os genótipos, sem exceção, quando comparados os resultados obtidos a zero e $24 \mathrm{mg} / \mathrm{L}$ de $\mathrm{Al}^{3+}$. O mesmo ocorreu quanto à característica CLF, com exceção dos híbridos H12 e H13. Elevada amplitude de variação foi observada para a relação RPAP (de $17,26 \%$, para o híbrido H13, até $72,35 \%$, para a 
TABELA3. Resultados médios obtidos para os caracteres altura de plântula (AP), comprimento de lâmina foliar (CLF) e redução porcentual de altura de plântula (RPAP) em 15 híbridos e três cultivares de capim-colonião, submetidos às concentrações de zero e $24 \mathrm{mg} / \mathrm{L}$ de $\mathrm{Al}^{3+}$, em soluções nutritivas, avaliados aos 45 dias $^{1}$.

\begin{tabular}{|c|c|c|c|c|c|}
\hline \multirow[t]{2}{*}{ Híbrido/cultivar } & \multicolumn{2}{|c|}{$\mathrm{AP}(\mathrm{cm})$} & \multicolumn{2}{|c|}{ CLF $(\mathrm{cm})$} & \multirow{2}{*}{$\begin{array}{c}\text { RPAP }^{2} \\
\%)\end{array}$} \\
\hline & $0 \mathrm{mg} / \mathrm{L}$ & $24 \mathrm{mg} / \mathrm{L}$ & $0 \mathrm{mg} / \mathrm{L}$ & $24 \mathrm{mg} / \mathrm{L}$ & \\
\hline $\mathrm{H} 10$ & $22,49 \mathrm{cde} A$ & $8,84 \mathrm{efgB}$ & 15,86 defA & $6,30 \mathrm{fghB}$ & $60,22 \mathrm{defg}$ \\
\hline $\mathrm{H} 12$ & $21,97 \mathrm{cde} A$ & $15,62 \mathrm{bcB}$ & $13,76 \mathrm{fgA}$ & $11,54 \mathrm{bcA}$ & $29,29 \mathrm{ab}$ \\
\hline H13 & $19,47 \mathrm{efA}$ & $16,13 \mathrm{bB}$ & $13,73 \mathrm{fgA}$ & $12,03 \mathrm{abA}$ & $17,26 \mathrm{a}$ \\
\hline $\mathrm{H} 21$ & $22,29 \mathrm{cdeA}$ & $10,64 \mathrm{deB}$ & $16,19 \operatorname{cdef} A$ & $8,00 \mathrm{cbB}$ & $59,96 \mathrm{cdef}$ \\
\hline $\mathrm{H} 22$ & $13,91 \mathrm{hA}$ & $6,20 \mathrm{ghB}$ & $11,01 \mathrm{hiA}$ & $4,43 \mathrm{hB}$ & $55,52 \mathrm{cdefg}$ \\
\hline H31 & $15,20 \mathrm{gh} A$ & $5,33 \mathrm{hB}$ & $11,88 \mathrm{ghA}$ & $4,05 \mathrm{hB}$ & $64,84 \mathrm{efg}$ \\
\hline H33 & $14,65 \mathrm{hA}$ & 8,44 efghB & $9,36 \mathrm{iA}$ & $6,53 \mathrm{fghB}$ & $42,15 \mathrm{bcd}$ \\
\hline H38 & $20,12 \operatorname{def} A$ & $10,14 \mathrm{defB}$ & $14,86 \mathrm{efA}$ & $8,64 \mathrm{defB}$ & $48,90 \mathrm{cde}$ \\
\hline H42 & $18,04 \mathrm{fgA}$ & $10,18 \mathrm{defB}$ & $18,71 \mathrm{abcA}$ & 7,74efB & $43,41 \mathrm{bcd}$ \\
\hline H54 & $26,67 \mathrm{aA}$ & $19,23 \mathrm{aB}$ & $20,52 \mathrm{aA}$ & $14,28 \mathrm{aB}$ & $27,99 \mathrm{ab}$ \\
\hline $\mathrm{H} 55$ & $24,12 \mathrm{abcA}$ & 9,93 defB & $17,05 \mathrm{bcde} A$ & $7,31 \mathrm{efgB}$ & $58,70 \mathrm{cdefg}$ \\
\hline H56 & $24,26 a b c A$ & $12,60 \mathrm{cdB}$ & $18,49 \mathrm{abcA}$ & $9,17 \mathrm{cdeB}$ & $48,04 \mathrm{cde}$ \\
\hline H64 & $23,04 \mathrm{bcdA}$ & $12,88 \mathrm{cdB}$ & $17,82 \mathrm{bcdA}$ & $9,55 \mathrm{cdeB}$ & $44,04 \mathrm{bcd}$ \\
\hline H79 & $25,80 \mathrm{abA}$ & $20,66 \mathrm{aB}$ & $18,90 \mathrm{abA}$ & $2,43 \mathrm{abB}$ & $18,90 \mathrm{a}$ \\
\hline $\mathrm{H} 140$ & $22,51 \mathrm{cdeA}$ & $6,91 \mathrm{fhgB}$ & $18,95 \mathrm{abA}$ & $5,18 \mathrm{ghB}$ & $69,31 \mathrm{fg}$ \\
\hline IAC-Centauro & $21,84 \mathrm{cde} A$ & $6,08 \mathrm{ghB}$ & 15,34 defA & 5,06 ghiB & $72,35 \mathrm{~g}$ \\
\hline IAC-Centenário & $20,55 \operatorname{def} A$ & $14,68 \mathrm{bcB}$ & $14,31 \mathrm{fgA}$ & $10,73 \mathrm{bcdB}$ & $28,48 \mathrm{ab}$ \\
\hline IAC-Tobiatã & $20,69 \operatorname{def} A$ & $11,42 \mathrm{deB}$ & $15,82 \mathrm{def} A$ & $8,55 \mathrm{efgB}$ & $40,28 b c$ \\
\hline Média & $20,98 \mathrm{~A}$ & $11,43 \mathrm{~B}$ & $15,70 \mathrm{~A}$ & $8,42 \mathrm{~B}$ & 46,04 \\
\hline
\end{tabular}

${ }^{1}$ Médias, seguidas de letras minúsculas diferentes, na mesma coluna, e de letras maiúsculas diferentes, na mesma linha, diferem significativamente entre si, a $\mathrm{P}=0,05$, de acordo com o teste de Duncan; coeficiente de variação de AP, CLF e RPAP igual a 11,20, 11,84 e 14,04\%, respectivamente.

${ }^{2}$ Dados transformados em $\operatorname{arcsen} \sqrt{\%}$.

TABELA 4. Resultados médios obtidos para os caracteres comprimento total (CTR) e líquido de raiz (CLR) e redução porcentual de comprimento líquido de raiz (RPCLR) em 15 híbridos e três cultivares de capim-colonião, submetidos às concentrações de zero e $24 \mathrm{mg} / \mathrm{L} \mathrm{de} \mathrm{Al}^{3+}$, em soluções nutritivas, avaliados aos 45 dias $^{1}$.

\begin{tabular}{|c|c|c|c|c|c|}
\hline \multirow[t]{2}{*}{ Híbrido/cultivar } & \multicolumn{2}{|c|}{ CTR $(\mathrm{cm})$} & \multicolumn{2}{|c|}{ CLR $(\mathrm{cm})$} & \multirow{2}{*}{$\frac{\text { RPCLR }^{2}}{(\%)}$} \\
\hline & $0 \mathrm{mg} / \mathrm{L}$ & $24 \mathrm{mg} / \mathrm{L}$ & $0 \mathrm{mg} / \mathrm{L}$ & $24 \mathrm{mg} / \mathrm{L}$ & \\
\hline $\mathrm{H} 10$ & $30,23 \mathrm{hA}$ & $11,67 \mathrm{ghB}$ & $25,05 \mathrm{gA}$ & $6,39 \mathrm{fgB}$ & $74,68 \mathrm{de}$ \\
\hline H12 & $30,60 \mathrm{ghA}$ & $20,93 \mathrm{cdeB}$ & $25,50 \mathrm{fgA}$ & $15,92 \mathrm{cdB}$ & $37,42 \mathrm{a}$ \\
\hline H13 & 36,66 defA & $17,93 \mathrm{efB}$ & $31,40 \mathrm{cdeA}$ & $12,62 \mathrm{deB}$ & $59,56 b c$ \\
\hline $\mathrm{H} 21$ & $55,29 \mathrm{aA}$ & $15,98 \mathrm{fgB}$ & $49,09 \mathrm{aA}$ & $9,63 \mathrm{efB}$ & $80,10 \mathrm{e}$ \\
\hline $\mathrm{H} 22$ & $24,87 \mathrm{iA}$ & $9,33 \mathrm{hiB}$ & $18,71 \mathrm{hA}$ & $3,12 \mathrm{ghB}$ & $83,19 \mathrm{ef}$ \\
\hline $\mathrm{H} 31$ & $23,27 \mathrm{iA}$ & $10,95 \mathrm{hB}$ & $17,54 \mathrm{hA}$ & $5,14 \mathrm{fghB}$ & $69,82 \mathrm{cde}$ \\
\hline $\mathrm{H} 33$ & $23,74 \mathrm{iA}$ & 7,48hiB & $17,71 \mathrm{hA}$ & $1,46 \mathrm{ghB}$ & $91,84 \mathrm{fg}$ \\
\hline H38 & $51,23 \mathrm{abA}$ & $23,48 \mathrm{bcdB}$ & $45,44 \mathrm{abA}$ & $17,47 \mathrm{bcdB}$ & $61,01 \mathrm{~cd}$ \\
\hline $\mathrm{H} 42$ & 37,11cdefA & $19,11 \mathrm{defB}$ & $31,32 \mathrm{cdeA}$ & $13,80 \mathrm{deB}$ & $55,90 \mathrm{bc}$ \\
\hline H54 & 33,24 fghA & $21,71 \mathrm{cdeB}$ & $27,01 \mathrm{efg} \mathrm{A}$ & $15,02 \mathrm{cdB}$ & $44,38 \mathrm{ab}$ \\
\hline H55 & $35,45 \mathrm{defg} \mathrm{A}$ & $23,48 \mathrm{bcdB}$ & 29,08 defgA & $17,37 \mathrm{bcdB}$ & $39,82 \mathrm{a}$ \\
\hline H56 & $41,91 \mathrm{cA}$ & $27,57 \mathrm{abB}$ & $36,11 \mathrm{cA}$ & $21,88 \mathrm{abB}$ & $39,30 \mathrm{a}$ \\
\hline H64 & $47,48 \mathrm{bA}$ & $30,85 \mathrm{aB}$ & $41,68 \mathrm{bA}$ & $25,12 \mathrm{aB}$ & $39,87 \mathrm{a}$ \\
\hline $\mathrm{H} 79$ & $39,86 \mathrm{cdA}$ & $24,15 \mathrm{bcB}$ & $32,94 \mathrm{cdA}$ & $17,70 \mathrm{bcdB}$ & $44,71 \mathrm{ab}$ \\
\hline H140 & 39,70 cdeA & 8,20hiB & $33,75 \mathrm{cdA}$ & $2,60 \mathrm{ghB}$ & $92,31 \mathrm{fg}$ \\
\hline IAC-Centauro & 34,72 efghA & $4,59 \mathrm{iB}$ & $28,95 \mathrm{defgA}$ & $0,95 \mathrm{hB}$ & $97,02 \mathrm{~g}$ \\
\hline IAC-Centenário & 37,21 cdefA & $25,47 \mathrm{bcB}$ & $30,66 \mathrm{def} A$ & $19,22 \mathrm{bcB}$ & $37,19 \mathrm{a}$ \\
\hline IAC-Tobiatã & 34,09 fghA & $10,18 \mathrm{hB}$ & $27,31 \mathrm{efgA}$ & $3,56 \mathrm{ghB}$ & $81,59 \mathrm{e}$ \\
\hline Média & $36,48 \mathrm{~A}$ & $17,39 \mathrm{~B}$ & $30,51 \mathrm{~A}$ & $11,61 \mathrm{~B}$ & 62,76 \\
\hline
\end{tabular}

\footnotetext{
${ }^{1}$ Médias, seguidas de letras minúsculas diferentes, na mesma coluna, e de letras maiúsculas diferentes, na mesma linha, diferem significativamente entre si, a $\mathrm{P}=0,05$, de acordo com o teste de Duncan; coeficiente de variação de CTR, CLR e RPCLR igual a 10,68, 14,70 e 9,31\%, respectivamente.

${ }^{2}$ Dados transformados em $\operatorname{arcsen} \sqrt{\%}$.
} 
cultivar IAC-Centauro), confirmando a existência de comportamento diferencial da parte aérea dos genótipos quanto ao efeito prejudicial do $\mathrm{Al}^{3+}$.

Também neste caso foram detectadas reduções significativas de CTR e CLR em todos os híbridos e cultivares em teste, quando comparados os resultados obtidos a zero e $24 \mathrm{mg} / \mathrm{L} \mathrm{de} \mathrm{Al}^{3+}$. Quanto à relação RPCLR, sobressaíram-se os híbridos H12, H54, H55, H64, H79 e a cultivar IAC-Centenário. A amplitude de variação foi também elevada (de 37,19\%, para a cultivar IAC-Centenário, até $97,02 \%$, para a cultivar IAC-Centauro).

A análise de componentes principais, realizada nas seis características descritas (AP, CLF, RPAP, CTR, CLR e RPCLR), originou um diagrama de dispersão, apresentado na Fig. 1. O eixo x do mesmo abrangeu $79,2 \%$ da variância total disponível enquanto o eixo y foi responsável por $16,7 \%$ da variabilidade estimada; assim, a variância total, expressa no diagrama, atingiu um alto nível $(95,9 \%)$, proporcionando uma excelente representatividade dos genótipos em estudo (Cruz \& Regazzi, 1994).

Com base no diagrama de dispersão, quatro classes de reação ao $\mathrm{Al}^{3+}$ puderam ser identificadas (localização de um dado genótipo quanto às relações RPAPe RPCLR): a) resistência: baixas RAP e RPCLR (híbridos H12, H13, H54, H79 e cultivar IAC-Centenário); b) tolerância: baixa RPAP e alta RPCLR (híbrido H33 e cultivar IAC-Tobiatã); c) sensibilidade: alta RPAP e baixa RPCLR (híbridos H38, H42, H55, H56 e H64); d) suscetibilidade: altas RPAPe RPCLR (híbridos H10, H21, H22, H31, H140 e cultivar IAC-Centauro).

As classificações das cultivares IAC-Centauro e IAC-Centenário, obtidas neste trabalho (suscetível e resistente, respectivamente), são idênticas às reações ao $\mathrm{Al}^{3+}$ já determinadas anteriormente para as mesmas cultivares. Já a classificação obtida para o

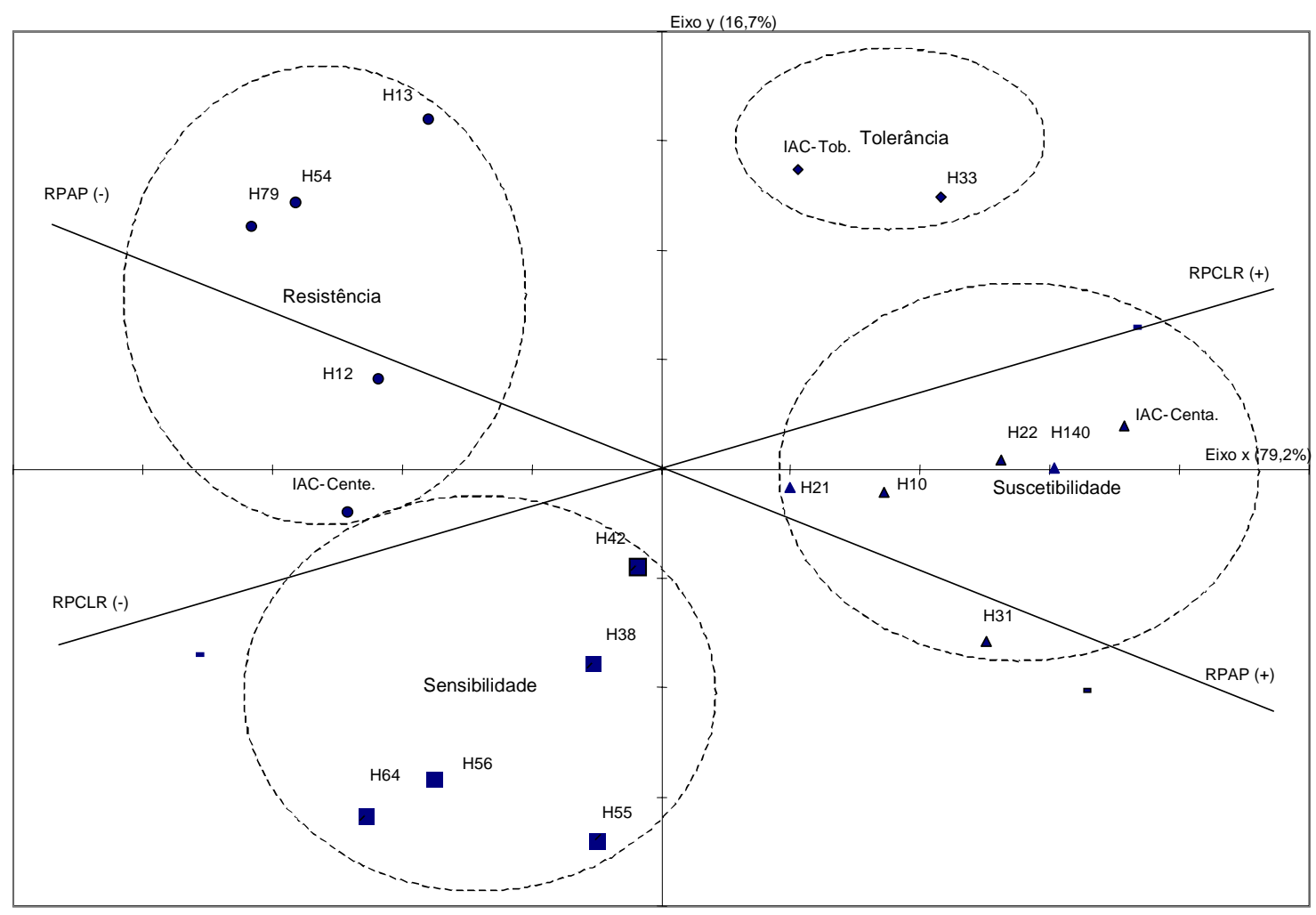

FIG. 1. Diagrama de dispersão e classificação de reação ao alumínio de 15 híbridos e três cultivares de capimcolonião. RPAP: redução porcentual de altura de plântula; RPCLR: redução porcentual de comprimento líquido de raiz. 
IAC-Tobiatã (tolerante) diverge da obtida anteriormente (moderadamente resistente). A razão parece ser que, na presente pesquisa, os materiais genéticos foram classificados com base em análise conjunta de reduções porcentuais de características de parte aérea e de sistema radicular, muito mais precisa do que a análise anterior apenas do crescimento radicular frente ao $\mathrm{Al}^{3+}$. Para confirmar ou não a classificação estudada, foi realizada análise fatorial discriminante (AFD). Esta detectou que os genótipos, sugeridos como pertencentes às quatro classes de reação ao $\mathrm{Al}$, foram agrupados com 94,3\% de acerto.

O sistema proposto para classificação de reação frente ao estresse de $\mathrm{Al}^{3+}$ é inédito em capim-colonião e mesmo em outras espécies de elevado potencial econômico. Baseado no isolamento do efeito do $\mathrm{Al}^{3+}$ em características de parte aérea e do sistema radicular, em solução nutritiva, seguido de análise de componentes principais, é extremamente importante para o melhoramento genético da espécie, além de ser muito útil na tomada de decisões quanto ao correto manejo de pastagens melhoradas.

Além disso, considerando-se cada uma das concentrações de $\mathrm{Al}^{3+}$ empregadas como um ambiente distinto, procedeu-se a análise de estabilidade e adaptabilidade fenotípicas (modelo de Eberhart \& Russell, 1996) para a característica altura de planta (AP), bom indicador do potencial forrageiro dos materiais genéticos em estudo. O modelo citado é o mais adequado quando da ocorrência de reduzido número de ambientes em avaliação (Vencovsky \& Barriga, 1992).

Os coeficientes de regressão linear ( $\beta_{1 i}$ 's), que são estimativas de resposta de cada genótipo às variações ambientais (Vencovsky \& Barriga, 1992), diferenciaram os materiais genéticos quanto à reação ao $\mathrm{Al}^{3+}$, com amplitude de variação de 0,3354 (H13) até 1,6515 (IAC-Centauro) (Tabela 5). Quando $\beta_{1 \mathrm{i}}$ é estatisticamente menor que 1,0 (híbridos H12, H13, H33, H79 e cultivar IAC-Centenário) existem fortes indícios de ocorrência de baixa sensibilidade às alterações ambientais presentes, isto é, maior adaptação

TABELA 5. Estimativas de parâmetros de estabilidade e adaptabilidade fenotípicas para o caráter altura de plântula de híbridos e cultivares de capim-colonião, submetidos a três ambientes (concentrações de zero, 12 e $24 \mathrm{mg} / \mathrm{L}$ de $\mathrm{Al}^{3+}$, em solução nutritiva), por 45 dias'.

\begin{tabular}{lcccc}
\hline Híbrido/cultivar & $\beta_{0 \mathrm{i}}$ & $\beta_{1 \mathrm{i}}$ & $\sigma_{\mathrm{di}}^{2}$ & $\begin{array}{c}\mathrm{R}_{\mathrm{i}}^{2} \\
(\%)\end{array}$ \\
\hline H12 & & & 84,88 \\
H13 & 17,78 & $0,6968^{*}$ & $2,9185^{\mathrm{ns}}$ & 85,83 \\
H33 & 18,07 & $0,3354^{* *}$ & $0,2081^{\mathrm{ns}}$ & 99,04 \\
H79 & 11,17 & $0,6576^{*}$ & $0,8704^{\mathrm{ns}}$ & 89,03 \\
IAC-Centenário & 23,70 & $0,5252^{* *}$ & $0,5020^{\mathrm{ns}}$ & 95,91 \\
H55 & 17,78 & $0,6018^{*}$ & $0,3531^{\mathrm{ns}}$ & 99,36 \\
IAC-Centauro & 16,91 & $1,4743^{* *}$ & $0,4206^{\mathrm{ns}}$ & 99,50 \\
H21 & 13,47 & $1,6515^{* *}$ & $1,0631^{\mathrm{ns}}$ & 97,42 \\
H22 & 15,54 & $1,2423^{\mathrm{ns}}$ & $0,8141^{\mathrm{ns}}$ & 91,31 \\
H31 & 9,86 & $0,8060^{\mathrm{ns}}$ & $1,0486^{\mathrm{ns}}$ & 91,97 \\
H38 & 10,82 & $1,0013^{\mathrm{ns}}$ & $3,3168^{*}$ & 95,93 \\
H42 & 13,93 & $1,0798^{\mathrm{ns}}$ & $3,6255^{*}$ & 91,62 \\
H54 & 14,33 & $0,8059^{\mathrm{ns}}$ & $0,2046^{\mathrm{ns}}$ & 98,15 \\
H56 & 23,35 & $0,7552^{\mathrm{ns}}$ & $1,3365^{\mathrm{ns}}$ & 94,00 \\
H64 & 17,59 & $1,2400^{\mathrm{ns}}$ & $0,2691^{\mathrm{ns}}$ & 99,26 \\
IAC-Tobiatã & 16,87 & $1,0941^{\mathrm{ns}}$ & $2,4554^{\mathrm{ns}}$ & 93,43 \\
H10 & 16,00 & $0,9625^{\mathrm{ns}}$ & $0,7472^{\mathrm{ns}}$ & 95,39 \\
H140 & 14,15 & $1,4720^{* *}$ & $5,9534^{*}$ & $4,6059^{*}$ \\
\hline
\end{tabular}

$1 \beta_{0 \mathrm{i}}:$ média referente aos três ambientes (concentrações de zero, 12 e $24 \mathrm{mg} / \mathrm{L}$ de $\mathrm{Al}^{3+}$ ); média geral: 15,$91 ; \mathrm{R}_{\mathrm{i}}^{2}:$ coeficiente de determinação.

ns, * e ** Não-significativo e significativo a $5 \%$ e $1 \%$ de probabilidade, respectivamente, pelo teste $\mathrm{t}$ (Student) para o coeficiente de regressão linear $\left(\beta_{1 \mathrm{i}}\right)$ e pelo teste $\mathrm{F}$ para as variâncias dos desvios da regressão $\left(\sigma_{\mathrm{di}}^{2}\right)$. 
a altas concentrações de $\mathrm{Al}^{3+}$ (Moda-Cirino, 1984). Siqueira (1997) enfatiza que, na seleção entre genótipos, devem ser escolhidos aqueles com maiores médias fenotípicas nos ambientes desfavoráveis (H12, H13, H79 e cultivar IAC-Centenário, Tabela 3). Por outro lado, os materiais genéticos com $\beta_{1 i}$ 's estatisticamente maiores que 1,0 são altamente afetados pela presença de concentrações crescentes de Al (baixa adaptabilidade) (H10, H55, H140, cultivar IAC-Centauro). Finalmente, aqueles apresentando $\beta_{1 i}$ 's estatisticamente iguais a 1,0 apresentam moderada capacidade de adaptação às condições ambientais vigentes, variável de acordo com o genótipo considerado.

Para os desvios de regressão linear ( $\sigma_{\mathrm{di}}^{2}$ 's), que estimam a previsibilidade de resposta dos materiais genéticos ao estresse, a grande maioria dos híbridos/cultivares em estudo revelou elevada previsibilidade de resposta quando da alteração das concentrações de $\mathrm{Al}^{3+}\left(\sigma_{\mathrm{di}}^{2}\right.$ 's estatisticamente iguais a zero). Apenas alguns deles (H10, H31, H38 e H140) mostraram comportamento instável frente ao estresse ( $\sigma_{\mathrm{di}}^{2}$ 's estatisticamente maiores que zero).

Finalmente, os coeficientes de determinação $\left(\mathrm{R}_{\dot{i}}^{2}\right.$ 's) estimados, variando de $84,88 \%$ a $99,51 \%$, atestam a elevada confiabilidade dos resultados obtidos.

Assim, os materiais genéticos puderam ser classificados em cinco grupos distintos, a saber: a) altas adaptabilidade e previsibilidade de resposta: H12, H13, H33, H79 e cultivar IAC-Centenário; b) moderada adaptabilidade e elevada previsibilidade de resposta: H21, H22, H42, H54, H56, H64 e cultivar IAC-Tobiatã; c) moderada adaptabilidade e baixa previsibilidade de resposta: H31 e H38; d) baixa adaptabilidade e elevada previsibilidade de resposta: H55 e cultivar IAC-Centauro; e) baixas adaptabilidade e previsibilidade de resposta: H10 e H140.

Comparando-se a classificação obtida através de análise de componentes principais com a previsibilidade de resposta ao estresse, observa-se que todos os materiais resistentes (H12, H13, H54, H79 e cultivar IAC-Centenário) e tolerantes (H33 e cultivar IAC-Tobiatã) apresentaram elevada previsibilidade de resposta; no grupo dos sensíveis, quatro dos cinco híbridos (80\%) - H42, H55, H56 e H64 - tiveram resposta previsível, o mesmo ocorrendo, no grupo dos suscetíveis, para três dos seis genótipos (50\%) - H21, H22 e cultivar IAC-Centauro. Considerando-se a elevada variabilidade presente, entre os híbridos em estudo, para diversos caracteres morfofisiológicos e mesmo para características de qualidade de sementes (Paterniani, 1996), pode-se concluir que o conjunto de metodologias empregado apresenta elevada chance de sucesso na discriminação de genótipos quanto à reação ao $\mathrm{Al}^{3+}$, o que sugere o seu emprego em programas de melhoramento genético voltados à resistência/tolerância ao estresse.

O emprego da associação de análises multivariada e de estabilidade e adaptabilidade fenotípicas como método de avaliação de genótipos quanto à reação diferencial ao $\mathrm{Al}^{3+}$ é inédito. Entretanto, análises de estabilidade e adaptabilidade fenotípicas (Hernani, 1980; Moda-Cirino,1984) ou de análise multivariada (Braccini et al., 1998) já foram utilizadas, isoladamente, com o mesmo objetivo.

\section{CONCLUSÕES}

1. A metodologia desenvolvida é eficiente e confiável como sistema de classificação de reação de genótipos ao estresse de alumínio.

2. A metodologia é também aplicável em experimentação de campo com o capim-colonião bem como com outras espécies vegetais, tanto em relação ao estresse de alumínio como de outros agentes estressantes.

\section{REFERÊNCIAS}

BASTOS, C.R. Toxicity of aluminum in nutrient solution of sorghum seedlings. Starkville : Mississippi State University, 1981.57p. M.Sc. Thesis.

BRACCINI, M.C.L.; MARTINEZ, H.E.P.; PEREIRA, P.R.G.; SAMPAIO, N.F.; SILVA, E.A.M. Tolerância de genótipos de cafeeiro ao alumínio em solução nutritiva. I. Crescimento e desenvolvimento da parte aérea e sistema radicular. Revista Brasileira de Ciência do Solo, Campinas, v.22, n.3, p.435-442, 1998. 
CAMARGO, C.E.O.; OLIVEIRA, O.F. Tolerância de cultivares de trigo a diferentes níveis de alumínio em solução nutritiva e no solo. Bragantia, Campinas, v.40, n.1, p.21-31, 1981.

CRUZ, C.D.; REGAZZI, A.J. Modelos biométricos aplicados ao melhoramento genético. Viçosa : UFV, 1994. 390p.

EBERHART, S.A.; RUSSELL, W.A. Stability parameters for comparing varieties. Crop Science, Madison, v.6, n.1, p.36-40, 1966.

FOY, C.D. Physiological effects of hydrogen, aluminum and manganese toxicities in acid soil. In: ADAMS, F. (Ed.). Soil acidity and liming. Madison: American Society of Agronomy, 1984. p.57-97.

FOY, C.D. Plant adaptation to mineral stress in problem soils. Iowa State Journal of Research, Ames, v.57, n.3, p.339-354, 1983.

FURLANI, P.R.; CLARK, R.B. Screening sorghum for aluminum tolerance in nutrient solution. Agronomy Journal, Madison, v.73, n.4, p.587-594, 1981.

HERNANI, L.C. Metodologia de seleção de cultivares de feijão (Phaseolus vulgaris L.) tolerantes ao alumínio em solução nutritiva. Piracicaba : ESALQ, 1980. 81p. Dissertação de Mestrado.

HUTTON, E.M. Breeding acid-soil tolerant lines of the tropical grass Panicum maximum Jacq. In: INTERNATIONAL GRASSLAND CONGRESS, 16., Nice, 1989. Proceedings. Nice : Association Française pour la Production Fourragere, 1989. p.355-356.

LAFEVER, H.N. Genetic differences in plant responses to soil nutrient stress. Journal of Plant Nutrition, New York, v.4, n.1, p.89-109, 1981.

McCORMICK, L.H.; STEINER, K.C. Variation in aluminum tolerance among six genera of trees: toxicity, surface mine revegetation. Forest Science, Bethesda, v.24, n.4, p.565-568, 1978.
McLEAN, F.T.; GILBERT, B.E. The relative aluminum tolerance of crop plants. Soil Science, Baltimore, v.24, n.2, p.163-176, 1927.

MODA-CIRINO, V. Reação de genótipos de arroz (Oryza sativa $\mathrm{L}$.) de sequeiro ao alumínio em solução nutritiva. Piracicaba : ESALQ, 1984. 162p. Dissertação de Mestrado.

NOWAK, J.; FRIEND, A.L. Aluminum sensitivity of loblolly pine and slash pine seedlings grown in solution culture. Tree Physiology, Victoria, v.15, n.9, p.605-609, 1995.

PATERNIANI, R.S. Avaliação de caracteres morfológicos, de qualidade de sementes e suas correlações em híbridos inéditos de Panicum maximum Jacq. Campinas : Unicamp, 1996. 67p. Dissertação de Mestrado.

SIQUEIRA, W.S. Desempenho agronômico e estabilidade fenotípica de clones de alho (Allium sativum L.) derivados do cultivo in vitro. Campinas : Unicamp, 1997. 135p. Tese de Doutorado.

SIVAGURU, M.; PALIWAL, K. Differential aluminum tolerance in some tropical rice cultivars. I. Growth performance. Journal of Plant Nutrition, New York, v.16, n.9, p.1705-1716, 1993.

STEEL, R.G.D.; TORRIE, J.H. Principles and procedures of statistics. Tokyo : McGraw-Hill, 1980. 633p.

THOMAS, D.; LAPOINTE, S. Testing new accessions of guinea grass (Panicum maximum Jacq.) for acid-soils and resistance to spittlebug (Aeneolamia reducta). Tropical Grasslands, St. Lucia, v.23, n.4, p.232239, 1989.

VENCOVSKY, R.; BARRIGA, P. Genética biométrica no fitomelhoramento. Ribeirão Preto : Sociedade Brasileira de Genética, 1992. 496p. 